\title{
Hipercoagulabilidad y daño endotelial en pacientes con enfermedad pulmonar obstructiva crónica en fase estable
}

\section{Hypercoagulability state and endotelial injury in stable chronic obstructive pulmonary disease patients}

\author{
M. A. Aibar ${ }^{1}$, K. Laborda ${ }^{2}$, F. Conget ${ }^{3}$, R. Cornudella ${ }^{4}$
}

\section{RESUMEN}

La enfermedad pulmonar obstructiva crónica es en la actualidad la cuarta causa de muerte en nuestro país. El principal agente causal de la enfermedad es el tabaco, cuyos efectos sobre el árbol bronquial no se limitan al parénquima pulmonar sino que además lesionan la pared endotelial, lo que podría contribuir a la aparición de trombosis, isquemia o hipertensión pulmonar.

En nuestro trabajo se estudia la existencia de un estado protrombótico en estos pacientes caracterizado por la activación de la coagulación y por el daño endotelial y, para ello, se comparan las cifras de marcadores bioquímicos en enfermos con EPOC y en sujetos sanos.

Se seleccionaron 51 pacientes con EPOC y un grupo de 30 voluntarios sanos de edades similares y se compararon las cifras de fibrinógeno, D-dímero, factor VIII y factor von Willebrand (FvW:Ag y FvW:Rico).

Se observó un aumento de todos los marcadores en el grupo de pacientes con respecto al grupo de controles sanos. Las diferencias encontradas fueron estadísticamente significativas en todos los casos $(\mathrm{p}<0,05)$.

La EPOC se acompaña de un estado de activación de la coagulación y de un daño endotelial que podrían contribuir al desarrollo de complicaciones vasculares como trombosis, isquemia o hipertensión pulmonar.

Palabras clave. Hipercoagulabilidad. Daño endotelial. EPOC.

\begin{abstract}
COPD is currently the fourth cause of death in our country; the main causal agent of the disease is tobacco whose effects on the bronchial tree are not limited to the lung parenchyma. The action of tobacco injures the endothelial wall, which could contribute to lung thrombosis, ischemic events or secondary pulmonary hypertension.

Our work investigates the existence of a prothombotic state in these patients, characterized by the activation of clotting and endothelial injury, comparing the values of biochemical markers between COPD patients and healthy volunteers.

Fifty-one patients with COPD and a group of 30 healthy volunteers of similar ages were selected and fibrinogen, D-dimer, factor VIII and von Willebrand factor (FvW:Ag and FvW:Rico) levels were compared.

We found an increase of all markers in the patient group compared to the healthy control group. The differences found were statistically significant in all cases $(\mathrm{p}<0.05)$.

COPD is characterized by a state of hypercoagulability and endothelial injury that they could contribute to the development of vascular complications such as lung thrombosis, ischemic events or secondary pulmonary hypertension.
\end{abstract}

Key words. Hypercoagulability state. Endothelial injury. COPD.
1. Servicio de Medicina Interna. Hospital Clínico Universitario Lozano Blesa. Zaragoza.

2. Centro de Salud Delicias Norte. Zaragoza.

3. Servicio de Neumología. Hospital Clínico Universitario Lozano Blesa. Zaragoza.

4. Servicio de Hematología y Hemoterapia. Hospital Clínico Universitario Lozano Blesa. Zaragoza.

Recepción: 2 de noviembre de 2009

Aceptación provisional: 15 de diciembre de 2009

Aceptación definitiva: 22 de enero de 2010

\section{Correspondencia}

Miguel Ángel Aibar Arregui

Tarazona, $7-3^{\circ} \mathrm{E}$

50017 Zaragoza

Tfno. 661016273

E-mail: miguel_a_a_a@hotmail.com

Los autores no declaran ningún conflicto de intereses ni financiación externa para la realización de este trabajo. 


\section{INTRODUCCIÓN}

La enfermedad pulmonar obstructiva crónica (EPOC) es una de las enfermedades crónicas más importantes en todos los países industrializados. Su prevalencia ha ido en aumento en los últimos años y en la actualidad supone un problema sociosanitario de primer orden tanto por su elevada mortalidad (cuarta causa de muerte en España) como por el enorme coste económico derivado de su diagnóstico y tratamiento ${ }^{1-3}$.

A pesar de la importancia objetiva de esta enfermedad y del conocimiento actual sobre su diagnóstico y tratamiento, el estudio IBERPOC demostró una situación de infradiagnóstico y unas diferencias de prevalencia entre las distintas áreas geográficas que obligan a plantearse si la EPOC está recibiendo la atención sanitaria que merece y si los programas para su prevención están alcanzando la efectividad necesaria ${ }^{3}$.

Desde el punto de vista asistencial, es importante considerar que el $60 \%$ de las consultas de estos pacientes son atribuibles a un grupo reducido de ellos (12,2\%) por su mayor gravedad, edad o situación social $^{4}$.

Las previsiones para el futuro son que la EPOC pasará a ser la tercera causa de muerte en los próximos años; además, la cronicidad del proceso y la mejora de los tratamientos supondrán un aumento considerable de su prevalencia y por lo tanto de los recursos sanitarios destinados a ella.

En este contexto, es crucial delimitar los factores que condicionan el pronóstico de la enfermedad y localizar grupos de pacientes con mayor demanda asistencial para poder controlar tanto el impacto social de la enfermedad como el económico.

Los principales factores pronósticos en la EPOC son el abandono del hábito tabáquico, la edad del paciente, la gravedad de la obstrucción y el desarrollo de complicaciones como la hipertensión pulmonar (HP) $)^{1,3,5}$.

Cuando hablamos de esta complicación en concreto hay que considerar que la fisiopatología de la HP es compleja y de- pende de la interacción de factores como la hipoxia, la alteración de la respuesta vasodilatadora, la aparición de trombosis in situ y el remodelado vascular del endotelio.

En este sentido, la alteración del equilibrio entre sustancias vasodilatadoras y vasoconstrictoras, la alteración de la agregación plaquetaria y la trombosis in situ se encuentran presentes en la HP de cualquier origen $^{5,6}$.

En los pacientes con EPOC, diferentes autores han estudiado la existencia de alteraciones de la coagulación y de lesiones endoteliales que podrían contribuir al desarrollo de la hipertensión pulmonar, aunque los resultados de los diferentes estudios han sido dispares y en la mayoría de los casos no está claro si estas alteraciones son la causa o la consecuencia de la $\mathrm{HP}^{7-9}$.

En el presente trabajo se estudian algunas alteraciones de la coagulación en los pacientes con EPOC y la existencia de un daño endotelial que puede contribuir al desarrollo de complicaciones vasculares y a empeorar el pronóstico de esta enfermedad.

\section{MATERIAL Y MÉTODOS}

Los pacientes incluidos en este estudio fueron seleccionados en el Servicio de Neumología del Hospital Clínico Universitario de Zaragoza, tanto en la consulta como en la planta de hospitalización. Mediante la revisión completa de su historia clínica se descartó la existencia de factores que pudieran alterar los resultados de coagulación, tales como la toma de anticoagulantes o antiagregantes, presencia de enfermedades hematológicas, neoplasias y enfermedades digestivas.

Una vez incluidos en el estudio se aseguró un periodo mínimo de estabilidad clínica de dos meses, con objeto de que los valores bioquímicos fueran basales y no en el curso de una agudización.

Un total de 51 pacientes fueron incluidos en el estudio y, una vez cumplidos estos dos meses de estabilidad clínica, se les extrajo una muestra de sangre para la 
determinación de fibrinógeno, D-dímero, factor VIII y factor von Willebrand (FvW:Ag y FvW:Rico).

Los sujetos del grupo control se seleccionaron entre los donantes de sangre que acuden con regularidad a nuestro hospital. Mediante entrevista personal se comprobaron los antecedentes médicos y terapéuticos de estos voluntarios con objeto de excluir factores que pudiesen alterar los resultados y, a continuación, se realizó el mismo estudio de coagulación que en los pacientes con EPOC tras confirmar un periodo similar sin donaciones de sangre ni enfermedades activas. Los sujetos del grupo control que eran fumadores fueron estudiados mediante espirometría para descartar la existencia de una EPOC no diagnosticada.

Los valores de fibrinógeno se determinaron por nefelometría mediante el sistema ACL $3000{ }^{\circledR}$ (IL) en muestras de plasma recogidas en tubos de citrato sódico; se consideran normales valores entre $200 \mathrm{y}$ $450 \mathrm{mg} / \mathrm{dl}$.

El Factor VIII se cuantificó mediante el sistema ACL $9000 \AA$ (IL), basado en la prueba del tiempo de tromboplastina parcial activado. Este método expresa los resultados en porcentaje con un rango de normalidad entre 60 y $120 \%{ }^{10}$.

La determinación cuantitativa del antígeno del factor von Willebrand (FvW:Ag), se realizó mediante un test inmunológico de aglutinación en látex. La aglutinación producida por la reacción entre el plasma y los anticuerpos utilizados en la técnica es directamente proporcional a la concentración del FvW:Ag. La medición de esta reacción se realiza por turbidimetría. La cuantificación de la actividad del factor von Willebrand (FvW:Rico) se realizó con esta misma técnica. Para ambos parámetros se consideran valores dentro de la normalidad los comprendidos entre 50 y $150 \%{ }^{11}$.

El Dímero-D se cuantificó mediante inmunoturbidimetría con partículas intensificadoras usando el TurbiTime System ${ }^{\circledR}$. Las proteínas contenidas en el suero humano forman inmunocomplejos con anticuerpos específicos. El enturbiamiento producido después de la mezcla se mide fotométricamente y se consideran valores normales los comprendidos entre 0 y $500 \mu \mathrm{g} / \mathrm{l}^{12}$.

Todos los datos de los pacientes se archivaron en una base de datos de Access ${ }^{\circledR}$ y desde allí se exportaron a las hojas de cálculo Excel $^{\circledR}$ y al paquete estadístico SPSS $11.5^{\circledR}$ para su análisis.

Mediante la prueba de KolmogorovSmirnov se descartó la distribución normal de las diferentes variables. Para el cálculo de diferencias significativas se utilizó la prueba estadística de la U de Mann-Whitney. Se consideraron relevantes niveles de significación del 95\% $(\mathrm{p}<0,05)$.

Todos los participantes en el estudio fueron informados verbalmente sobre la realización del mismo aceptando su inclusión.

\section{RESULTADOS}

Completaron el estudio un total de 51 pacientes diagnosticados de EPOC, 50 de ellos varones $(98,03 \%)$ y una mujer $(1,96 \%)$. La edad media del grupo fue de 63,92 \pm 5,9 años con un rango que osciló entre los 46 años del paciente más joven y los 70 del mayor.

En lo que se refiere al consumo de tabaco, 14 pacientes $(27,45 \%)$ eran fumadores activos en el momento de ser incluidos en el estudio y 37 eran exfumadores (72,54\%). El consumo medio de tabaco fue de 70,29 \pm 34,69 paquetes por año (rango 4-135).

Atendiendo a la clasificación de la iniciativa $\mathrm{GOLD}^{1}$, 16 pacientes presentaban una EPOC moderada (31,3\%), 24 grave $(47,0 \%)$ y 11 muy grave $(21,5 \%)$. El FEV me- $_{1}$ dio fue de $42,4 \pm 14,7 \%$ y el cociente $\mathrm{FEV}_{1} /$ FVC de $52 \pm 10,7 \%$.

En este grupo, 13 pacientes (25,49\%) estaban en tratamiento con oxigenoterapia continua domiciliaria (OCD), otros 3 con OCD y BIPAP $(5,88 \%)$, mientras que el resto (35 pacientes; $68,62 \%$ ) no precisaba o no utilizaba estos tratamientos por diferentes razones. De los pacientes con OCD, 5 seguían fumando $(31,2 \%)$. 
En lo que se refiere a su situación gasométrica, el 47,05\% (24) presentaban hipoxemia y otros $20(39,21 \%)$ insuficiencia respiratoria (9 insuficiencia respiratoria global); tan sólo 7 pacientes $(13,72 \%)$ presentaban cifras de $\mathrm{PaO}_{2}$ superiores a $80 \mathrm{mmHg}$ en reposo y respirando aire ambiente. En total, 14 pacientes $(27,45 \%)$ presentaban cifras de $\mathrm{PaCO}_{2}$ iguales o superiores a $45 \mathrm{mmHg}$.
El estudio de coagulación ofreció los resultados que se muestran en la tabla 1; tan sólo 2 pacientes presentaban cifras elevadas de factor VIII y ninguno de FvW:Ag o FvW:Rico. El fibrinógeno estaba elevado en 24 pacientes $(47,05 \%)$ y el D-Dímero en 6 (sólo 3 de ellos tenían elevado también el fibrinógeno).

Tabla 1. Diferencias entre los valores medios del grupo de EPOC (I) y el grupo control (II).

\begin{tabular}{lllc}
\hline & $\begin{array}{c}\text { Grupo I } \\
(\mathbf{n = 5 1 )}\end{array}$ & \multicolumn{1}{c}{$\begin{array}{c}\text { Grupo II } \\
\mathbf{( n = 3 0 )}\end{array}$} & $\begin{array}{c}\text { U } \\
\text { Mann-Whitney }\end{array}$ \\
\hline D-Dímero* & $339,34 \mu \mathrm{g} / \mathrm{l}$ & $244,70 \mu \mathrm{g} / \mathrm{l}$ & 0,009 \\
Fibrinógeno & $469,71 \mathrm{mg} / \mathrm{dl}$ & $334,67 \mathrm{mg} / \mathrm{dl}$ & 0,000 \\
Factor VIII & $113,73 \%$ & $105,13 \%$ & 0,011 \\
FvW: Ag & $113,76 \%$ & $110,87 \%$ & 0,015 \\
FvW: Rico & $105,92 \%$ & $98,83 \%$ & 0,004 \\
\hline
\end{tabular}

${ }^{*}$ Se han suprimido los datos de un paciente (grupo I) por su disparidad con el resto de parámetros.

Cabe destacar los valores extremos de dímero-D detectados en un paciente con cifras de $4.200 \mu \mathrm{g} / \mathrm{l}$, a pesar de encontrarse en fase estable y sin sintomatología clínica, sus cifras de fibrinógeno, FVIII y FvW eran normales. Para el análisis estadístico se han suprimido las cifras correspondientes al D-dímero de este paciente sin que se haya podido determinar la causa de esta alteración a pesar de su estudio.

El grupo control se formó con voluntarios sanos, seleccionados entre los donantes de sangre de nuestra área sanitaria. De los 30 sujetos incluidos 29 eran varones (96,66 \%), y una mujer (3,33\%). La edad media fue de 62,1 $\pm 4,7$ años (rango 52-70).

En el momento de incorporarse al estudio $8(26,66 \%)$ eran fumadores, $11(36,66 \%)$ exfumadores y los otros 11 (36,66 \%) no habían fumado nunca. La media de consumo de tabaco fue de $17,73 \pm 23,10$ paquetes por año para el grupo completo y de $28 \pm 23,6$ si sólo consideramos a los fumadores (rango 5-90).

Se realizaron espirometrías a 7 de los 8 fumadores del grupo control que resultaron normales para su edad y sexo; el otro participante se negó a su realización y, por lo tanto, a pesar de que no presentaba clínica respiratoria alguna no puede descartarse por completo la presencia de una EPOC.

Sólo un individuo de este grupo tenía elevada la cifra de D-Dímero $(585 \mu \mathrm{g} / \mathrm{l})$. Además, otro voluntario presentó cifras elevadas de fibrinógeno. Ninguno de los otros parámetros apareció alterado en los sujetos del grupo control.

En la tabla 1 se muestran los valores medios de los diferentes datos de coagulación obtenidos para el grupo de pacientes EPOC y para el grupo de controles sanos así como la significación estadística encontrada utilizando la prueba de Mann-Withney. Las diferencias encontradas fueron estadísticamente significativas en todos los casos, demostrándose valores más altos en el grupo de pacientes que en el grupo control.

\section{DISCUSIÓN}

La EPOC es en la actualidad la cuarta causa de muerte en nuestro país, y lo esperado es que se convierta en la tercera en 
los próximos años. En los últimos años, se han publicado cifras de prevalencia, informaciones sobre el mal seguimiento de la enfermedad y estudios de impacto económico que nos deben obligar a reflexionar sobre la importancia que se le concede en el día a día ${ }^{2-4}$.

Uno de los aspectos más importantes a considerar es el pronóstico de la enfermedad; como es sabido, el abandono del hábito tabáquico y el tratamiento con oxigenoterapia continua domiciliaria (OCD) son los únicos factores capaces de modificar su curso natural, pero existen otros factores cuyo conocimiento puede contribuir a este objetivo. Uno de estos factores es la hipertensión pulmonar (HP) cuyo estudio ha crecido en interés gracias al desarrollo de nuevas estrategias terapéuticas, hasta el punto de que su clasificación se ha modificado dos veces en los últimos años ${ }^{13-14}$.

En la patogenia de la HP intervienen diferentes factores y, aunque no está claro si son causa o consecuencia del desarrollo de la HP, las alteraciones de la coagulación y el daño endotelial que la acompañan han sido estudiados por diferentes autores en los pacientes con EPOC e incluso se ha estudiado si el tratamiento ambulatorio con heparinas de bajo peso molecular de forma profiláctica podría reducir el número de complicaciones y la mortalidad en estos pacientes aunque por el momento los resultados no avalan su uso ${ }^{15}$.

El principal objetivo de nuestro estudio es valorar la existencia de un estado protrombótico en los pacientes con EPOC en fase estable mediante la determinación de marcadores de hipercoagulabilidad y de daño endotelial; estas alteraciones podrían contribuir al desarrollo de complicaciones vasculares tales como la hipertensión pulmonar o la embolia de pulmón empeorando el pronóstico de la EPOC por lo que la actuación profiláctica y terapéutica sobre ellas debería convertirse en uno de nuestros objetivos en el futuro.

Para ello, uno de los marcadores usados en nuestro trabajo es el fibrinógeno, proteína soluble que participa en la formación del trombo. En los últimos años gran cantidad de estudios han identificado al fibrinógeno como un importante factor de riesgo cardiovascular implicado tanto en el desarrollo de aterosclerosis como de enfermedad coronaria $^{16,17}$ en lo que se refiere a la EPOC; diferentes autores han coincidido en señalar que incluso en fases estables la concentración de fibrinógeno está elevada en los pacientes con respecto a los sujetos sanos $7,18,19$.

Autores como Welsh han señalado la influencia del fibrinógeno en el desarrollo y mantenimiento de la hipertensión pulmonar que, como es sabido, acompaña con frecuencia a las fases avanzadas de la EPOC $^{20}$ aunque en nuestro grupo un $47 \%$ de los pacientes tenían elevadas las cifras de fibrinógeno demostrando diferencias muy significativas con respecto al grupo control, no hemos encontrado correlación con la gravedad de la obstrucción ni con otros indicadores de progresión de la enfermedad, sin duda por el reducido tamaño muestral de nuestro estudio; estos datos aparecen reflejados en anteriores trabajos de los autores ${ }^{18}$.

Otro de los marcadores usados en nuestro trabajo es el dímero-D, producto de degradación específico de la fibrina cuya elevación refleja un aumento del turn-over de esta proteína que se produce con la edad, los procesos inflamatorios, las neoplasias $\mathrm{y}$, en general, con cualquier situación que active la coagulación ${ }^{21}$.

La elevación de los niveles de dímeroD se utiliza habitualmente como screening del tromboembolismo de pulmón (TEP), en particular para descartar su existencia en pacientes con bajo riesgo de $\mathrm{TEP}^{17,22}$, sin embargo, sus valores pueden estar elevados en presencia de microtrombos que no produzcan un TEP significativo pero que a la larga sí podrían contribuir al desarrollo de trombosis o de $\mathrm{HP}^{23,24}$.

En el 11,7\% de nuestros pacientes se encontró una elevación del D-Dímero por encima del rango de la normalidad así como una diferencia significativa con los valores medios de los sujetos sanos, la elevación de los niveles de esta marcador en los pacientes con EPOC ya ha sido reportada por otros autores? 
El tercero de los marcadores utilizados en nuestro estudio fue el factor von Willebrand (FvW), glucoproteína sintetizada tanto por las células endoteliales como por los megacariocitos. En la actualidad pueden cuantificarse los niveles circulantes de FvW de dos formas diferentes, midiendo su actividad antigénica (FvW:Ag) o su actividad biológica (FvW:Rico). La actividad antigénica traduce la concentración total de la molécula, mientras que la actividad biológica traduce su capacidad funcional y se acepta que este factor puede secretarse al torrente sanguíneo en respuesta al daño endotelial de cualquier origen ${ }^{25,26}$.

Aunque en nuestro grupo ninguno de los pacientes presenta cifras fuera del rango de normalidad, sí que hay una diferencia significativa entre los valores medios de los pacientes y los de sujetos sanos; una posible explicación es la amplitud del rango de normalidad en la técnica de laboratorio utilizada. Estas diferencias se encuentran tanto al estudiar la cantidad de FvW como su capacidad funcional lo que se debe, según diversos autores, a la producción de moléculas defectuosas a consecuencia del daño endotelial presente en estos pacientes ${ }^{20,27}$.

En pacientes con EPOC, se ha demostrado un aumento del FvW circulante, aunque esta elevación no se ha relacionado con un mayor descenso del $\mathrm{FEV}_{1}$, mientras que algunos autores correlacionan de forma satisfactoria los niveles de FvW:Ag con las cifras de presión en arteria pulmonar. Estos datos avalan la idea de que el daño endotelial juega un importante papel en el desarrollo de HP en las fases avanzadas de la EPOC ${ }^{27,28}$.

Por último, hemos estudiado las concentraciones de factor VIII, proteína hemostática que acelera la activación del factor X cuya elevación se considera, además de un signo de daño endotelial, un factor de riesgo trombótico y predictivo para el desarrollo de eventos cardiacos ${ }^{29}$.

En nuestra revisión no hemos encontrado artículos que valoren la elevación del factor VIII en el curso de la EPOC, aunque es conocido el hecho de que este factor se eleva en los fumadores con respecto a los sujetos sanos ${ }^{30}$.
En otro de nuestros trabajos se ha valorado la elevación encontrada en los niveles de factor VIII en los pacientes con EPOC respecto a controles sanos y su posible relación con parámetros objetivos de gravedad de la enfermedad, encontrando correlaciones significativas con datos gasométricos y espirométricos ${ }^{18}$. Diferentes autores han relacionado la elevación del factor VIII con el TEP y con la hipertensión pulmonar de origen tromboembólico por lo que todos estos datos invitan a pensar que esta elevación, reflejo del daño endotelial, podría estar en el origen de la HP y otras complicaciones vasculares en los pacientes con $\mathrm{EPOC}^{31-33}$.

Considerando en conjunto todos los marcadores estudiados $\mathrm{y}$, a pesar de las limitaciones de nuestro estudio, la principal de las cuales es sin duda el reducido tamaño muestral alcanzado, nuestros resultados orientan hacia la existencia de alteraciones tanto en la coagulación como a nivel endotelial que deberían ser estudiadas con mayor profundidad para concretar su importancia en la evolución de la enfermedad.

Para confirmar estas alteraciones sería preciso un estudio prospectivo que contribuyera a resolver las limitaciones que suponen el reducido tamaño muestral y la escasa incorporación de mujeres a la muestra.

\section{BIBLIOGRAFÍA}

1. Pauwels RA, Buist AS, Ma P, Jenkins CR, Hurd SS. Global strategy for the diagnosis, management, and prevention of chronic obstructive pulmonary disease: National Heart, Lung, and Blood Institute and World Health Organization global Initiative for chronic obstructive lung disease (GOLD): executive summary. Respir Care 2001; 46: 798-825.

2. KRIEF B. Impacto social y económico de la EPOC en España. Farmacoeconomía 1996; 5: 8-19.

3. Soriano JB, Ancochea J, Miravitlles M, GarcíaRío F, Duran E, MuÑoz L et al. Recent trends in COPD prevalence in Spain: a repeated crosssectional survey 1997-2007. Eur Respir J 2009 Dec 8 [Epub ahead of print]. 
4. Soler JJ, Sánchez L, Latorre M, Alamar J, Román P, PERPIÑ́́ M. Impacto asistencial hospitalario de la EPOC. Peso específico del paciente con EPOC de alto consumo sanitario. Arch Bronconeumol 2001; 37: 375-381.

5. Higenbottam T. Pathophysiology of pulmonary hypertension; a role for endothelial dysfunction. Chest 1994; 105 (Suppl): 7S-12S.

6. Loscalzo J. Endothelial dysfunction in pulmonary hypertension. N Engl J Med 1992; 327: 117-119.

7. Alessandri C, Basili S, Violi S, Ferroni P, GazzaNIGA PP, Cordova C et al. Hypercoagulability state in patients with chronic obstructive pulmonary disease. Thromb Haemost 1994; 72: 343-346.

8. Peinado Vi, Pizarro S, Barberá JA. Pulmonary vascular involvement in COPD. Chest 2008; 134: 808-814.

9. Budhiraja R, Tuder R, Hassoun P. Endothelial dysfunction in pulmonary hypertension. Circulation 2004; 109: 159-165.

10. National committee for clinical laboratory standards. One stage prothrombin time (PT) test and activated partial thromboplastin time (APTT) test; Approved guideline, NCCLS Document H47-A, vol. 16 (3).

11. Newman D, Henneberry H, Price C. Particle enhanced light scattering immunoassay. Ann Clin Biochem 1992; 29: 22-42.

12. Metzmann E. Protein quantitation on both branches of the Heidelberg curve by monitoring the kinetic of immunoprecipitation. Behring institute mitteilungen 1985; 78: 167175.

13. Fishman A. Clinical classification of pulmonary hypertension. Clin Chest Med 2001; 22: 385-391.

14. Simonneau G, Galie N, Rubin L, Langleben D, SeeGer W, Domenighetti G et al. Clinical classification of pulmonary hypertension. J Am Coll Cardiol 2004; 43: 5S-12S.

15. Modesto M, Nauffal D, Ansótegui E, MenÉndez $\mathrm{R}$, Ballesta A, Touza $\mathrm{R}$ et al. Can home prophylaxis for venous thromboembolism reduce mortality rates in patients with chronic obstructive pulmonary disease? Arch Bronconeumol 2006 Mar; 42: 130-134.

16. KoenIG W. Fibrinogen in cardiovascular disease: an update. Thromb Haemost 2003; 89: 601-609.

17. Danish J, Lewington S, Thompson SG, Lowe GD, Collins R, Kostis JB et al. Fibrinogen Studies Collaboration. Fibrinogen level and the risk of major cardiovascular diseases and nonvascular mortality: an individual participant meta-analysis. JAMA 2005; 294: 1799-1809.

18. Aibar M.A. Estado protrombótico e hipertensión pulmonar en pacientes con EPOC [tesis doctoral] . Zaragoza: Universidad de Zaragoza, 2005.

19. Peter K. MacCallum. Markers of hemostasis and systemic inflammation in heart disease and atherosclerosis in smokers. Proc Am Thorac Soc 2005; 2: 34-43

20. Welsh CH, Hassell KL, Badesch DB, Kressin DC, MARLAR RA. Coagulation and fibrinolytic profiles in patients with severe pulmonary hypertension. Chest 1996; 110: 710-717.

21. Folsom A. Haemostatic risk factors for atherothrombotic disease: an epidemiologic view. Thromb Haemost 2001; 86: 366-373.

22. PARENT F, MAÎTRE S, MEYER G, R AHERISON C, MAL $\mathrm{H}$, LANCAR R et al. Diagnostic value of D-dimer in patients with suspected pulmonary embolism: results from a multicentre outcome study. Thromb Res 2007; 120: 195-200.

23. Katsumura Y, OHTSUBO K. Association between pulmonary microthromboembolism and coagulation variables in hypercoagulable states: an autopsy study. Respirology 1999 ; 4: 239-243.

24. McNEIL K, DUNNING J. Chronic thromboembolic pulmonary hypertension (CTEPH). Heart 2007; 93: 1152-1158.

25. Ramos F, Batlle J, López Borrasca A. Mecanismos de alteración funcional del factor von willebrand. Med Clin (Barc) 1989; 92: 588594.

26. WAGNER D, Bonfanti R. Von Willebrand factor and the endothelium. Mayo Clin Proc 1991; 66: 621-627.

27. Penny WF, Weinsten M, Salzman EW, Ware A. Correlation of circulating von Willebrand factor levels with cardiovascular hemodynamics. Circulation 1991; 83: 1630-1636.

28. Chambers DC, Boldy DA, Ayres JG. Chronic respiratory symptoms, von Willebrand factor and longitudinal decline in FEV1. Respir Med 1999; 93: 726-733.

29. Tracy RP, Arnold AM, Ettinger W, Fried L, MeiLANH E, SAVAGE P. The relationship of fibrinogen and factors VII and VIII to incident cardiovascular disease and death in the elderly. The results from the cardiovascular health study. Arterioscler Thomb 1999; 19: 1776-1783.

30. TAPSON VF. The role of smoking in coagulation and thromboembolism in chronic obstructi- 
ve pulmonary disease. Proc Am Thorac Soc 2005; 2: 71-77.

31. Bonderman D, Turecek PL, Jakowitsch J, Weltermann A, Adlbrecht C, Schneider B et al. High prevalence of elevated clotting factor VIII in chronic thromboembolic pulmonary hypertension. Thromb Haemost 2003; 90: 372-376.
32. LANG I, KERR K. Risk factors for chronic thromboembolic pulmonary hypertension. Proc Am Thorac Soc 2006; 3: 568-570.

33. Erkekol O, Ulu A, Numanoglu N, Akar N. High plasma levels of factor VIII: An important risk factor for isolated pulmonary embolism. Respirology 2006; 11: 70-74. 